\title{
A Study on a Multiplicity of Load Balancing Algorithms
}

\author{
Vidyaa Thulasiraman ${ }^{1}$ and G. Manikandan ${ }^{2}$ \\ ${ }^{1}$ Assistant Professor and Head, Department of Computer Science, \\ Government Arts and Science College for Women, Bargur, Tamil Nadu, India \\ ${ }^{2}$ Research Scholar, Department of Computer Science, Periyar University, Salem, Tamil Nadu, India \\ E-Mail: vidyaathulasi@gmail.com,mnjayan5@gmail.com
}

\begin{abstract}
Past days rulers and publics need to convey message their relatives or one place to somewhere else with the assistance of some correspondence channels like birds or individuals like present mailmen or post ladies and days are passing and development of data and innovation the communication quick and less expansive and present day interchanges totally through on the web. Day by day populaces development and innovation utilizing people groups heterogeneously expanding. So present days network is a critical part and significant assuming jobs in day by day life its outcomes more system issues like less throughput, insufficient network resources, less flags, resource not similarly shared for a given timeframe, time complicity because of this troubles the communication mostly or here and there totally interfered with now daily's a fundamental piece of information is an expected portion to social and economic related change and it is logically expanding worldwide condition. In this exploration essentially an undertaking has been made to classify secured sharing models, architecture, sharing arrangements and load adjusting algorithms in w3. At present need to configure modern load adjusting algorithms on account of our central and state Government focusing propelled correspondences and modernized classrooms through distributed computing. Keywords: Load Adjusting, Performance, Algorithm
\end{abstract}

\section{INTRODUCTION}

Exceptional advancement in technology innovation has led to the ascent of the interest of fast handling and the need of high versatility, accessibility and quick reaction. This brought about utilization of similar and circulated registering frameworks where additional than one workstation forms the movement at the same time. One of the principle look into issues in parallel and dispersed framework is powerful procedure to circulate remaining task at hand among various processors. Burden adjusting is utilized for limiting the reaction time, expanding the throughput, and to stay away from the over-burden. Burden adjusting is to guarantee that each processor in the framework does around a similar measure of work anytime of time [1].

\section{A. Load Balancing}

Load matching helps to share out load crossways one or else more resource and also keep track of status of all resource while distributing resource request. If a server is not available it stops sending traffic. Burden adjusting is the procedure of circulation or redistribution of burden among processor subsequently improving the execution of the framework. In registering, load adjusting conveys outstanding burden over numerous figuring assets, for example, personal computer, a personal computer bunch, arrange connections, or focal handling units. Burden adjusting means to upgrade asset use, amplify throughput, limit response time, and maintain away from overstrain of any single resource [3].

\section{Types of Load Balancing}

a. Hardware Load Balancing: It is based on the hardware which works as load balancer but is very expansive, even big companies use them only as first point of contact and use other mechanism for load balancing.

b. Software Load Balancing: It is based on the hybrid approach in this approach every client request on this port will be received by proxy and then passed to the backend service in efficient way. Haproxy is the popular open source software.

\section{Necessitate of Load Balancing}

A conveyed framework contain amount of processors functioning freely with one another plus connected by correspondence control. A few be not connected with any correspondence channel. Every work station have an underlying burden with the purpose of is the measure of work to be performed, and each may have an alternate preparing limit. The work load have to be in order circulated among all processors dependent going on their handling alacrity with the goal that opportunity to execute all assignments gets limited and inactive time of every processor can be diminished. This is the reason we need load adjusting. Burden inequity is likewise a fundamental issue within information similar application moreover at these times additionally it chiefly happens because of the uneven circulation of information among the different processors in the framework. Without great burden circulation systems and procedures, we can't plan to achieve great speedup and great effectiveness.

\section{Issues Identified with Load Balancing}

The accompanying issues are broke down amid burden adjusting. 
a. During circulated condition the message channel be of limited data transmission and the handling unit might be alive actually inaccessible subsequently weight adjusting could do with to get choice of whether close to permit errand movement or else not.

b. A compute work might not discretionarily detachable prompting certain limitations in separating undertakings.

c. Each work comprises of a few littler assignments and every one of folk's errands is able to contain distinctive finishing period.

d. The load on every processor just as on the system can differ now and again dependent on the outstanding task at hand achieved by the clients.

e. The processors limit might be not the same as one another in engineering, activity framework, CPU speed, memory estimate, and accessible circle space.

Contemplating above elements the heap adjusting can be summed up into four Fundamental Advances:

1. Monitor workstation burden as well as situation

2. Exchange burden as well as circumstance data flanked by processors

3. Calculate the innovative job dispersion actual information group

\section{Policies of Load Balancing}

A decent burden adjusting calculation is characterized by some fundamental approaches [4]which are as per the following:

a. Information Policy: Specifies what remaining load of data to be gathered, refreshed, as soon as it is to be gathered and beginning anywhere.

b. Trigger rule: It determines the approximate time to time begin a heap adjusting activity.

c. Resource type rule: It is regain the assignment beginning the majority over-burden source or else not.

d. Situation policy: It is use the consequences of the source style approach on the way to locate an appropriate accomplice for a beneficiary

e. Choice policy: It define the errands which ought to be there relocated since busiest resources to most assemble resources. A determination strategy thinks about a few factors in choosing an assignment, for example exchange of little assignment will receive less overhead.

\section{Compensation of Load Balancing}

A few significant favorable circumstances of burden adjusting [5] are as per the following

a. It diminishes the assignment holding up time.

b. It limits errand reaction moment.

c. It maximizes use of framework resource.

d. It expand system throughput.

e. It improves consistency, and solidness of the framework. f. It obliges potential alteration.

g. Lengthy hunger is stayed away from for little job.

h. During weight adjusting generally speaking framework execution is upgrade by improving the execution of every hub. Due to the above advantages, the heap adjusting methodologies turn into a field of serious research. Therefore, an extensive number of planning and burden adjusting calculations have been created in the previous quite a while.

\section{LOAD COMPLEMENTARY APPROACH}

During burden adjusting nearby two methodologies and every methodology contain their very individual calculations. The two methodologies are as for every the following:

A. Static Load Balancing: Within the Static burden adjusting advance, the heap adjusting choices are complete deterministically otherwise probabilistically on accumulate moment as indicated by the execution of processing hubs as well as stay steady amid runtime. Number of errands in every hub be permanent in this methodology Fixed load adjust strategies be non-preemptive for example when the heap is distributed near the hub it insincerity be exchanged to one more hub.

Pros: It has least correspondence delay, Algorithms be basic as well as simple to actualize, and classification transparency is limited.

Cons: Assignment can't be relocated even as execution, on the whole execution of framework diminished because of burden variances, less helpful when assignment has diverse completing time as well as hubs is diverse.

1. Algorithms of Static Load Balancing: Amongst different projected calculations significant fixed burden adjusting calculations are as per the following:

a. Round Robin Algorithm: Round Robin Algorithm relegates assignments consecutively and uniformly to every one of the hubs. All tasks are doled out to processing hubs dependent on Round Robin request, implying that registering hubs picking is performed in arrangement and will have returned to the primary figuring hub if the last registering hub has been come to [6]. Every hub keeps up its heap file locally free of allotments from remote hub.

Pros: Inter-process correspondence isn't required, Useful for employments of equivalent preparing instance as well as hubs of similar abilities.

Cons: Not helpful while tasks have uneven handling instance, not helpful while hubs contain distinctive limits.

b. Randomized Algorithm: Randomized Algorithm utilizes arbitrary facts during choosing registering hubs for handling, with no have a few data about the present or past burden lying on the hub. The compute hubs are chosen 
arbitrarily subsequent arbitrary numbers created dependent lying on a measurement conveyance [8].

Pros: It functions admirably for specific unique reason applications, No bury process correspondence is required.

Cons: It isn't viewed as exquisite arrangement, Maximum reaction instance amongst everyone calculation.

c. Central Manager Algorithm: During every progression in middle Manager Algorithm focal hub chooses slave hub to be allocated an errand Slave hub having least burden is being chosen. The focal hub keeps up the heap list of all slave hubs associated with it. At whatever point, weight is distorted, a communication is send by the slave hubs to the focal hub. The heap chief settles on burden adjusting choices dependent on the framework load data, permitting the best choice when of the procedure made[5,1].

Pros: Perform healthy while active exercises are made by various hosts.

Cons: It desires abnormal state of bury method correspondence, Single purpose of disappointment.

d. Threshold Algorithm: During Threshold Algorithm undertakings or forms be appointed promptly winning formation to the figuring hubs (processors). Computing hubs designed for novel procedures are chosen in the neighborhood with no transfer inaccessible communication. Every hub keeps a confidential duplicate of the framework's heap data. The heap of a figure hub can be portrayed by one of the three dimensions which are: under stacked medium and over-burden. Two limit parametert_under and t_upperbe able to be utilized to portray these dimensions.

\section{i. $\quad$ Under stacked- work burden <t_under, \\ ii. Medium -t_under $\leq$ work burden $\leq t \_$upper, \\ iii. Overloaded-work burden >t_upper.}

In the underlying stage, all the registering hubs are considered under stacked. At whatever point the heap condition of a processing hub surpasses the heap level edge, at that point it sends messages with respect to the new burden state to the majority of the other registering hubs, consistently refreshing them so that as the real burden condition of the whole framework can be known to each hub. In the event that the neighborhood state isn't overburden, at that point the procedure is dispensed locally. Something else, an inaccessible beneath stacked workstation is chosen, and if rejection such multitude exists; the procedure is additionally dispensed in the neighborhood $[5,6]$.

Pros: It have low down inter process correspondence, presentation is better due to expansive amount of neighborhood progression distributions which diminishes the transparency of remote procedure allotments and isolated recollection gets to.

Cons: One over-burden workstation could comprise a lot higher burden than other over-burden processors, cause huge aggravation during burden adjusting, and enlarged completing point in time of are quest.

\section{B. Dynamic Load Balancing}

Present are three universal modules of issues everywhere a fixed burden adjusting is either unimaginable or can prompt irregularity of burden, issues are [2]:

1. The primary comprises of issues during which every one of the task is accessible toward the start of the calculation yet the measure of point in time necessary by each undertaking be unique.

2. The subsequent issues in which undertakings are accessible toward the start however as the calculation advances, the measure of point in time necessary by each errand change.

3. The last comprises of issues during which errands be not accessible toward the start but rather are created progressively. In static burden adjusting an excess of data about assignment and framework be compulsory prior to execution which is absurd each time like in these three classes of issues. So self-motivated burden adjusting is created to address these imperatives. Active burden adjusting settles on increasingly instructive burden adjusting choices amid execution by the runtime state data. In unique burden adjusting calculations outstanding task at hand is dispersed in the middle of the processors at runtime. These calculations screen change lying on the framework remaining task at hand and redistribute the work in like manner.

Pros

i. Dynamic burden adjusting functions admirably for heterogeneous frameworks.

ii. Assignment is able to be redistributing to a few workstation at the same time as run time henceforth over-burdening and under stacking issues end up least.

iii. It functions admirably for undertaking having distinctive execution time.

$i v$. The framework need not know about run-time conduct of the application earlier than finishing.

Cons

i. $\quad$ Prominent correspondence over heads happens and turns out to be more when number of processors increment.

ii. Dynamic burden adjusting calculations are unpredictable henceforth not simple to execute.

iii. System overhead increments since it are preemptive.

\section{Categories of Dynamic Load Balancing}

Present are three fundamental powerful weight adjusting calculations which are like subsequent:

1. Centralized: Come close to simply one workstation act while the major or root director as well as it is decide 
how toward assign job of the hubs as well as rest of the nodes proceed as slaves [9].

2. Decentralized: This come up to everyone nodes in the method are involved in construction the load adjusting conclusion and each node is in charge in organization their own possessions.

3. Cooperative: This approach every weight judgment creates or has the duty to take out its own part of the assignment.

4. Non-Cooperative: This come close to every node is self-governing have independence more than its individual source arrangement that is decision be finished in parallel.

5. Adaptive: This approaches the decision gets addicted to deliberation past and present system presentation and are pretentious by earlier decision or change in the situation.

6. Non Adaptive: This parameter use in load corresponding stay behind the same in spite of systems past behavior.

7. Sender Initiated: Overcrowded nodes try to be in motion work towards below burdened nodes.

8. Receiver Initiated: Underneath overloaded nodes call for tasks toward be alive sent to them from nods with superior heaps.

9. Symmetrical Initiated: Both the beneath burdened while well because the overloaded nodes might commence load transfer.

\section{Dynamic Load Balancing Algorithm}

Present are three basic active load balancing algorithm which belike follows.

1. Central Queue Algorithm: Supplies innovative exercises and displeased demands in a cyclical first in first out line on the primary host $[8,1]$. Each new movement landing at the line administrator is embedded into the line. At that point, at whatever point a demand for a movement is gotten by the line administrator, it expels the primary action from the line and sends it to the requester. On the off chance that there are no prepared exercises in the line, the demand is cradled, until another movement is accessible. While a workstation load cascade beneath the limit, the nearby burden administrator sends a demand for another action in the direction of the main burden director. The focal burden supervisor answers the demand promptly if a prepared movement is found in the process-ask for line, or lines the demand until another action arrives. This is a concentrated started calculation and need high correspondence among hubs.

Pros: Each new activity arriving at the queue Cons: It needs towering communication nodes

2. Local Queue Algorithm: This calculation underpins entomb progression movement. The principle idea in neighborhood line calculation is fixed assignment of every one new procedure by means of procedure relocation started by the host when its heap falls under the predefined least number of prepared procedures (edge limit). At first, innovative procedures made taking place the principle have are apportioned on all under stacked hosts. Starting that point lying on, every one of the procedures made lying on the prime host and every supplementary host be allotted close by. While the neighborhood has got less than burden it asks for the exercises on or after the remote hosts. The isolated hosts than look into its nearby rundown for prepared exercises as well as analyzes the neighborhood amount of prepared exercises with the get number. On the off chance that the previous is more noteworthy than the last mentioned, at that point a portion of the movements are accepted on to the requestor have as well as get the affirmation as of the multitude[12].

Pros: Support Process migration

Cons: Lesser communication compare middle line algorithm

3. Least Connection Algorithm: This calculation chooses the heap conveyance based lying on associations present on a hub [12]. The heap balancer monitors the quantities of associations joined to every hub and chooses the hub with least number of associations for burden exchange. The number increments when another association is set up and diminishes when association completes otherwise moment in time out. Least relations strategic job best in conditions somewhere the hubs have comparative abilities. Burden unevenness might be caused when the undertakings are all of various lengths since Connection checking simply doesn't represent that situation great.

Pros: Nodes have similar capabilities

Cons: Each and every one process formed on the central multitude only

\section{QUALITATIVE PARAMETER}

1. Nature: It tells whether calculation is fixed or active.

2. Overhead: It is measure of outlay like inter progression communiqué relocation of errands and so on included while actualizing the calculation and ought to be least.

3. Resource Utilization: It advises whether the calculation can use every one of the resource ideally or else not implies less inert processors.

4. Process Migration: It tells while a framework determination moves its progression. The calculation is fit for choosing when it should create change of burden appropriation amid execution of procedure or else not.

5. Fault Tolerant: It tell whether the calculation be able to work persistently in occasion of disappointment or else not, execution of calculation be diminishing or not.

6. Response Time: It is time a dispersed framework utilizing a specific burden adjusting calculation is attractive to react and should survive less.

7. Waiting Time: It is the timeframe spent holding up in the prepared line and ought to be less.

8. Adaptability: It advises if calculation can adjust to evolving circumstances. 
TABLE I COMPARATIVE ANALYSIS OF LOAD BALANCING ALGORITHM

\begin{tabular}{|c|c|c|c|c|c|c|c|c|c|}
\hline \multirow{6}{*}{ Static } & Parameter & & & & $\vec{\Xi}$ & 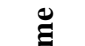 & $\mathscr{g}$ & $>\nabla$ & \\
\hline & Algorithms & 胥 & 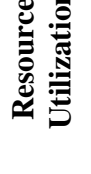 & 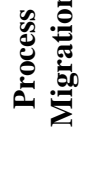 & 离 & 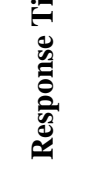 & 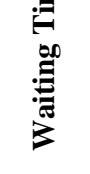 & 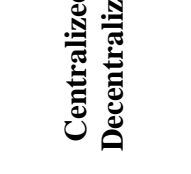 & 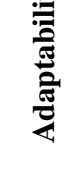 \\
\hline & Round Robin & Low & Less & No & No & Less & More & Decentralized & Less \\
\hline & Randomized & Low & Less & No & No & Less & More & Decentralized & Less \\
\hline & $\begin{array}{l}\text { Central } \\
\text { Manager }\end{array}$ & Low & Less & No & Yes & Least & More & Decentralized & Less \\
\hline & Threshold & High & Less & No & No & Less & More & Decentralized & Less \\
\hline \multirow{3}{*}{ Dynamic } & Central Queue & High & Less & No & Yes & More & Less & Centralized & More \\
\hline & Local Queue & High & More & Yes & Yes & More & Less & Centralized & More \\
\hline & $\begin{array}{l}\text { Least } \\
\text { Connection }\end{array}$ & High & More & No & No & Less & Less & Decentralized & More \\
\hline
\end{tabular}

TABLE II OUTLINE OF LOAD BALANCING ALGORITHMS

\begin{tabular}{|c|c|c|c|c|}
\hline Approaches & Algorithms & Key Features & Merits & Demerits \\
\hline \multirow{5}{*}{ Static } & Round Robin & $\begin{array}{l}\text { 1.Tasks are Sequentially } \\
\text { 2.The Nodes Serially }\end{array}$ & $\begin{array}{l}\text { 1.Refusal inter process } \\
\text { communiqué } \\
\text { 2.Simple to execute } \\
\text { 3.Less coordination } \\
\text { transparency }\end{array}$ & $\begin{array}{l}\text { 1.No helpful if nodes contain } \\
\text { dissimilar capacity } \\
\text { 2.Refusal functional if tasks } \\
\text { have untrustworthy running } \\
\text { time }\end{array}$ \\
\hline & $\begin{array}{l}\text { Weighted } \\
\text { Round Robin }\end{array}$ & $\begin{array}{l}\text { 1. Assigning tasks based } \\
\text { on their weights }\end{array}$ & $\begin{array}{l}\text { 1.Improvement over round } \\
\text { robin } \\
\text { 2.The nodes are different } \\
\text { capacities }\end{array}$ & $\begin{array}{l}\text { 1.There is no use if tasks } \\
\text { have different execution time }\end{array}$ \\
\hline & Randomized & $\begin{array}{l}\text { 1.Hubs are selected } \\
\text { randomly }\end{array}$ & $\begin{array}{l}\text { 1.No inter process } \\
\text { communication } \\
\text { 2.Helpful for special purpose } \\
\text { application }\end{array}$ & $\begin{array}{l}\text { 1.Response time is } \\
\text { maximum } \\
\text { 2.Load distribution uneven }\end{array}$ \\
\hline & $\begin{array}{l}\text { Central } \\
\text { Manager }\end{array}$ & $\begin{array}{l}\text { 1.Origin node select } \\
\text { slave nod } \\
\text { 2.Slightest load node is } \\
\text { elected }\end{array}$ & $\begin{array}{l}\text { 1.When dynamic tasks are } \\
\text { created it is useful }\end{array}$ & $\begin{array}{l}\text { 1.Inter process } \\
\text { communication is high } \\
\text { 2.Bottleneck state arises }\end{array}$ \\
\hline & Threshold & $\begin{array}{l}\text { 1.If load better doorstep } \\
\text { maximum task is assign }\end{array}$ & $\begin{array}{l}\text { 1. Inaccessible progression } \\
\text { allotment and recollection } \\
\text { contact is minimize }\end{array}$ & $\begin{array}{l}\text { 1. Completing time is greater } \\
\text { than before } \\
2 . \text { Load balancing is } \\
\text { disturbed }\end{array}$ \\
\hline \multirow{3}{*}{ Dynamic } & $\begin{array}{l}\text { Central } \\
\text { Queue }\end{array}$ & $\begin{array}{l}\text { 1. Stores new task in first } \\
\text { in first out } \\
\text { 2. Designation initiated } \\
\text { balancing }\end{array}$ & $\begin{array}{l}\text { 1. Helpful for heterogeneous } \\
\text { nodes } \\
\text { 2. After execution the tasks } \\
\text { assigned }\end{array}$ & $\begin{array}{l}\text { 1. Solo point of malfunction } \\
\text { 2. Not inter progression task } \\
\text { immigration }\end{array}$ \\
\hline & Local Queue & $\begin{array}{l}\text { 1. Inter progression task } \\
\text { movement if load fewer } \\
\text { than threshold edge }\end{array}$ & $\begin{array}{l}\text { 1. Inter process } \\
\text { communication is less }\end{array}$ & $\begin{array}{l}\text { 1. Algorithm supports inter } \\
\text { process migration }\end{array}$ \\
\hline & $\begin{array}{l}\text { Least } \\
\text { Connection }\end{array}$ & $\begin{array}{l}\text { 1. Load migrate to direct } \\
\text { to node with smallest } \\
\text { amount relations }\end{array}$ & $\begin{array}{l}\text { 1. It be helpful for nodes of } \\
\text { similar capabilities }\end{array}$ & $\begin{array}{l}\text { 1. No useful for different } \\
\text { task duration }\end{array}$ \\
\hline
\end{tabular}

\section{CONCLUSION}

In this paper we include taken survey of ideas regarding burden adjusting and different burden adjusting methodologies. Computing resource are quickly creating and developing jobs of varied framework with parallel or distributing processing issue of burden unevenness has risen and load adjusting is answer for such issue. At this time we displayed so as to heap adjusting circulates the heap equitably amongst hubs, thus expanding generally overall performance. At that point we contemplated different strategies which ought to survive consider even as planning the heap adjusting algorithm similar to information procedure and so forth. At last we examined Static and Dynamic burden offsetting systems with their individual calculations and inferred that every technique has their very 
own advantages and disadvantages and there exists no totally impeccable adjusting calculation yet one can utilize contingent upon the need.

\section{REFERENCES}

[1] Sandeep Sharma and Sarabjit Singh and Meenakshi Sharma, "Performance Analysis of Load Balancing Algorithms", World Academy of Science, Engineering and Technology, 2008.

[2] Mohsen and HosseinDelda, "Balancing Load in a Computational Grid Applying Adaptive", Intelligent Colonies of Ants Informatics, Vol. 32, pp. 327-335, 2008.

[3] ZhongXu and Rong Huang, "Performance Study of Load Balancing Algorithms in Distributed Web Server", World Academy of Science, Engineering and Technology,2008.

[4] G. R. Andrews and D. P. Dobkin, and P. J. Downey, "Distributed allocation with pools of servers," in ACMSIGACT-SIGOPS Symp.Principles of Distributed Computing, pp. 73-83,Aug. 1982.

[5] S. F. El-Zoghdy and S. Ghoniemy, "A Survey of Load Balancing In High-Performance Distributed Computing Systems", International Journal of Advanced Computing Research, Vol. 1, 2014.

[6] Daniel Grousa and T.Anthony, "Non-Cooperative load balancing in distributed systems", Journal of Parallel and Distributing Computing, 2005.

[7] P. A. Tijare and Dr. P. R. Deshmukh, "Schemes for Dynamic Load Balancing - A Review", International Journal of Advanced Research in Computer Science and Software Engineering, Vol.3, No. 6, pp. 688-69, June - 2013
[8] S.Amitkumar, Manekar, MukeshPoundekar, Prof. Hitesh Gupta and Prof. Malti Nagle, "A Pragmatic Study and Analysis of Load Balancing Techniques In Parallel Computing", International Journal of Engineering Research and Application, Vol. 2, No. 4, pp.19141918, July-August 2012.

[9] William Leinberger, George Karypis and Vipin Kumar, "Load Balancing Across Near-Homogeneous Multi-Resource Servers", 07695-0556- 2/00, 2000 IEEE.

[10] HendraRahmawan and YudiSatriaGondokaryono, "The Simulation of Static Load Balancing Algorithms",International Conference on Electrical Engineering and Informatics, Malaysia,2009.

[11] Y.Wang and R. Morris, "Load balancing in distributed systems," IEEE Trans Computing. Vol. C-34, No. 3, pp.204-217, Mar. 1985.

[12] AmitChhabra, Gurvinder Singh and Sandeep Singh Waraich, "Qualitative Parametric Comparison of Load Balancing Algorithms in Parallel and Distributed Computing Environment", World Academy of Science, Engineering and Technology, No. 16, pp.39-42, May 2006.

[13] Mr.Gaurav Sharma and JagjitKaur Bhatia, "A review on different approaches for load balancing in computational grid", Journal of Global Research in Computer Science, Vol. 4, No. 4, April 2013.

[14] R.L. Ribler, J.S. Vetter and H. Simitci, "Autopilot: adaptive Control of distributed applications",Proceedings of the Seventh IEEE Symposium on High-Performance Distributed Computing, pp. 172$179,1998$.

[15] ZhongXu, and Rong Huang, "Performance Study of Load Balancing Algorithms in Distributed Web Server Systems", CS213 Parallel and Distributed Processing Project Report. 